\title{
Effectiveness of Synthetic Polymer-coated Peripherally Inserted Central Catheter in Patients With Advanced Cancer
}

\author{
YUTA TAKEZAWA ${ }^{1,2}$, KOUJI IZUMI $^{2}$, TAIKI KAMIJIMA ${ }^{1}$, KAZUAKI MACHIOKA $^{1}$, \\ TAKASHI SHIMA ${ }^{1}$, HIROAKI IWAMOTO ${ }^{2}$, TAKAHIRO NOHARA ${ }^{2}$, \\ KAZUYOSHI SHIGEHARA ${ }^{2}$, YOSHIFUMI KADONO ${ }^{2}$, CHIKASHI SETO $^{1}$ and ATSUSHI MIZOKAMI ${ }^{2}$ \\ ${ }^{1}$ Department of Urology, Toyama Prefectural Central Hospital, Toyama, Japan; \\ ${ }^{2}$ Department of Integrative Cancer Therapy and Urology, Kanazawa University, \\ Graduate School of Medical Science, Kanazawa, Japan
}

\begin{abstract}
Background/Aim: A peripherally inserted central catheter (PICC) is recommended for the safe administration of anticancer agents. The effectiveness of synthetic polymercoated and non-coated PICCs was compared. Patients and Methods: Patients with advanced cancers who had indwelling PICCs were reviewed using their medical records. Three types of PICCs were compared in terms of complications and catheter failure. Results: A total of 90 patients were retrospectively analyzed, including 31 with Groshong PICCs, 30 with Argyle PICC kit, and 29 with Argyle PICC kit II. The incidence of catheter failure for Groshong PICC, Argyle PICC kit, and Argyle PICC kit II per 1,000 PICC days was 4.4614, 5.6617 , and 0.8658 , respectively. Catheter failure-free survival in the Argyle PICC kit II group was significantly better than that in the Argyle PICC kit group ( $p=0.0339)$. Conclusion: Argyle PICC kit II, a synthetic polymer-coated PICC, may render longer patency and prevention of catheter failure than non-coated PICCs.
\end{abstract}

Safe and accurate administration of anticancer agents is very important for the successful treatment of patients with advanced cancer who need intravenous chemotherapy (1). A peripherally inserted central catheter (PICC) via the basilic or cephalic vein is recommended to administer anticancer agents rather than a centrally inserted central catheter (CICC) that occasionally causes lethal mechanical complications (1,

This article is freely accessible online.

Correspondence to: Kouji Izumi, MD, Ph.D., Department of Integrative Cancer Therapy and Urology, Kanazawa University Graduate School of Medical Science, 13-1 Takara-machi, Kanazawa, Ishikawa 920-8641, Japan. Tel: +81 762652393, Fax: +81 762344263, e-mail: azuizu2003@yahoo.co.jp

Key Words: Peripherally inserted central catheter, advanced cancer, chemotherapy, catheter failure.
2). Complications sometimes also occur with PICCs; however, most complications are not lethal, such as venous thrombosis and infection, resulting in catheter failure and the need for catheter removal $(3,4)$. To avoid these problems, a synthetic polymer-coated PICC was developed and clinically applied for patients with advanced cancer; however, there is no study comparing the incidence of catheter failure between conventional non-coated and polymer-coated PICCs. In the present study, the effectiveness of the synthetic polymercoated PICC was assessed in terms of catheter patency and complications and compared with two types of conventional non-coated PICCs.

\section{Patients and Methods}

Study population. Patients with advanced cancer with indwelling PICCs who were seen at the Department of Urology, Kanazawa University Hospital between April 2014 and June 2017 were selected and retrospectively reviewed using their medical records. The present study was performed after receiving approval (No. 2016-320) from the Medical Ethics Committee of Kanazawa University.

Peripherally inserted central catheter (PICC). Three types of commercially available PICCs were included in the present study: Groshong PICC (Gr, Bard Access Systems, Salt Lake City, UT, USA), Argyle PICC kit (Ar1, Nippon Covidien, Tokyo, Japan), and Argyle PICC kit II (Ar2, Nippon Covidien and Toyobo, Osaka, Japan). Ar2 is coated with a synthetic polymer (SEC ONE COAT) to prevent platelet adhesion, protein adsorption, and complement activation. All PICCs were inserted via the basilic or cephalic vein under local anesthesia. We inserted Gr from April 2014 to April 6, 2015, and the catheter-inserted side was not fixed. We inserted Ar1 from April 14, 2015 to October 2015, either Ar1 or Ar2 from November 2015 to June 2016, and Ar2 after July 2016. After April 14,2015 , the right side was selected prior to the left side for catheter insertion to decrease complications $(5,6)$.

Definition and outcomes. The primary endpoint was catheter failurefree survival (CFFS), which was calculated from the date PICC was 
inserted to the date PICC was removed. Catheter failure was defined as removal due to complications. Complications that caused catheter failure were also examined, and the possibility of each complication per 1,000 PICC days (1000PDs) was calculated.

Statistical analysis. All statistical analyses were performed using commercially available GraphPad Prism software (GraphPad, San Diego, CA, USA). Comparisons among the three groups were made using one-way ANOVA and Chi-squared test. CFFS was estimated using the Kaplan-Meier method. Log-rank test was used for comparison of survival distributions. In all analyses, a $p$-value of $<0.05$ was considered statistically significant.

\section{Results}

Patient characteristics. The number of patients in Gr, Ar1, and Ar2 group was 31, 30, and 29, respectively. Although age, gender, and primary cancer site did not significantly differ among the three groups, the catheter-inserted side showed a significant difference (Table I). This difference reflected the difference in the policy for catheter insertion stated in the Patients and Methods section.

Events related to catheter. The median inserted period for $\mathrm{Gr}$, Ar1, and Ar2 group, and overall was 44 (range=6-147), 43 (range=6-176), 33 (range=4-112), and 41 (range=4-176) days, respectively. Although the proportion of patients who underwent intravenous chemotherapy though PICC and who underwent PICC port construction for outpatient administration of chemotherapy did not differ significantly among the three groups, the incidence of catheter failure in the Ar2 group was significantly lower than in the other groups (Table II).

Catheter complications resulting in failure. Four types of catheter complications resulting in failure were identified. The incidence of infection with or without luminal thrombus was higher in the Gr group $(n=5)$. In contrast, the incidence of other obstructions without infection and luminal thrombus was higher in the Ar1 group $(n=7)$. One spontaneous fracture of catheter was observed in the Gr group. The incidence of each complication per 1000PDs was calculated, and total incidence rate per 1000PDs in the Gr, Ar1, and Ar2 group was 4.4614, 5.6617, and 0.8658, respectively (Table III). Moreover, 30-day CFFS rate in the Gr, Ar1, and Ar2 group was $84.2 \%, 85.3 \%$, and $95.2 \%$, respectively. CFFS in the Ar2 group was significantly better than in the Ar1 group (Figure 1).

\section{Discussion}

Intravenous administration of anticancer agents is still the standard of care for treatment in patients with advanced cancer (1). Due to severe adverse effects, such as tissue destruction by
Table I. Patient characteristics.

\begin{tabular}{lcccc}
\hline Catheter & Gr $(\mathrm{n}=31)$ & Ar1 $(\mathrm{n}=30)$ & $\operatorname{Ar} 2(\mathrm{n}=29)$ & $p$-Value \\
\hline Median age (range) & $71(37-85)$ & $67(28-88)$ & $68(30-81)$ & 0.0607 \\
Gender & 8 & 6 & 8 & 0.7761 \\
$\quad$ Male & 23 & 24 & 21 & \\
Female & & & & \\
Side & 13 & 22 & 24 & 0.0022 \\
Right & 18 & 8 & 5 & \\
Left & & & & \\
Cancer & 18 & 16 & 13 & \\
Bladder and urethra & 6 & 5 & 2 & \\
Pelvis and Ureter & 5 & 0 & 3 & \\
Kidney & 0 & 2 & 2 & \\
Prostate & 1 & 3 & 5 & \\
Germ cell & 1 & 4 & 4 & \\
Others & & & & \\
\hline
\end{tabular}

Gr: Groshong PICC; Ar1: Argyle PICC kit; Ar2: Argyle PICC kit II.

Table II. Events related to catheter.

\begin{tabular}{lrrcc}
\hline & Gr $(\mathrm{n}=31)$ & $\operatorname{Ar} 1(\mathrm{n}=30)$ & $\operatorname{Ar} 2(\mathrm{n}=29)$ & $p$-Value \\
\hline IVCT & & & & \\
$\quad$ No & 7 & 2 & 6 & 0.1941 \\
$\quad$ Yes & 24 & 28 & 23 & \\
Port conversion & & & & \\
$\quad$ No & 30 & 28 & 26 & 0.5432 \\
$\quad$ Yes & 1 & 2 & 3 & \\
Failure & & & & \\
$\quad$ No & 24 & 22 & 28 & 0.0454 \\
$\quad$ Yes & 7 & 8 & 1 & \\
\hline
\end{tabular}

Gr: Groshong PICC; Ar1: Argyle PICC kit; Ar2: Argyle PICC kit II; IVCT: application of intravenous chemotherapy.

extravasation of vesicant and irritant agents, the administration of anticancer agents must be accurate and safe. Therefore, a central catheter is regarded as the best approach to administer these agents (2). Although CICC via subclavian vein was formerly a standard approach for intravenous hyperalimentation and chemotherapy, it sometimes resulted in unpredictable mechanical complications, including thoracentesis and arterial puncture (2). PICC was reported to have fewer mechanical complications related to the insertion procedure, and it has recently been recommended for administration of anticancer agents (1). Despite this advantage, PICC is associated with a higher risk of deep vein thrombosis compared with CICC $(3,7$, 8). To solve this problem, a synthetic polymer-coated PICC, Ar2, was developed.

In the present study, Ar2 showed not only significantly lower incidence of catheter failure than Gr and Ar1, but also showed significantly better CFFS than Ar1. This result 
Table III. Catheter complications resulting in failure.

\begin{tabular}{lcccccc}
\hline & $\mathrm{Gr}(\mathrm{n}=31)$ & $\operatorname{Ar} 1(\mathrm{n}=30)$ & $\operatorname{Ar} 2(\mathrm{n}=29)$ \\
\cline { 2 - 7 } & $\mathrm{n} / 1000 \mathrm{PDs}$ & $\mathrm{n}$ & /1000PDs & $\mathrm{n}$ & /1000PDs \\
\hline Infection without thrombus & 2 & 1.2747 & 1 & 0.7077 & 0 & 0 \\
Infection with thrombus & 3 & 1.9120 & 0 & 0 & 0 & 0 \\
Other obstructions & 1 & 0.6373 & 7 & 4.9540 & 1 & 0.8658 \\
Fracture & 1 & 0.6373 & 0 & 0 & 0 & 0 \\
Total & 7 & 4.4614 & 8 & 5.6617 & 1 & 0.8658 \\
\hline
\end{tabular}

Gr: Groshong PICC; Ar1: Argyle PICC kit; Ar2: Argyle PICC kit II; /1000PDs: per 1,000 PICC days.

confirms the direct benefit of coated synthetic polymer for Ar2. Although there was no significant difference in CFFS between $\mathrm{Gr}$ and $\mathrm{Ar} 2$, the incidence of complications per 1000PDs in $\operatorname{Ar} 2$ (0.8658) was one-fifth of $\mathrm{Gr}$ (4.4614). Ar2 may reduce the incidence of complications compared to $\mathrm{Gr}$. However, there was a significant difference in the side of PICC insertion between the Ar2 and Gr group. This difference may affect the incidence of complication, because the superiority of the right side insertion in terms of catheter complication has been reported $(5,6)$.

In the present study, the incidence of complications per 1000PDs in the Gr and Ar1 groups may be higher than previously reported $(1,9,10)$. As shown in Figure 1, the PICC-inserted period in some patients in the present study was relatively long (more than 50 days), and few complications occurred in these patients. Most catheter failure occurred within 30 days of insertion. Moreover, most PICCs were removed with no complication after a series of chemotherapy for urological malignancies, including neoadjuvant and adjuvant setting. Alternatively, the PICCinserted period in patients with hematological malignancies that required long-term chemotherapy was much longer (median 114.5 days) than that of the present study (median 41 days) (10). The fact that PICCs that were stably functioning were removed immediately after chemotherapy may be the reason for the relatively higher incidence of complications per 1000PDs in the present study.

The present study is the first to report that a synthetic polymer-coated PICC is safe and effective to administer anticancer chemotherapy with less catheter complications; however, the study has certain limitations. First, this was a retrospective study with a small sample size. Second, all patients were Japanese, and the primary site of cancer was almost exclusively genitourinary organs. The influence of the difference in ethnicity and chemotherapy protocol cannot be excluded. Finally, the diameter of PICC, PICCinserted vein, and patient background regarding hematological status that may influence thrombus

\section{Catheter failure-free survival}

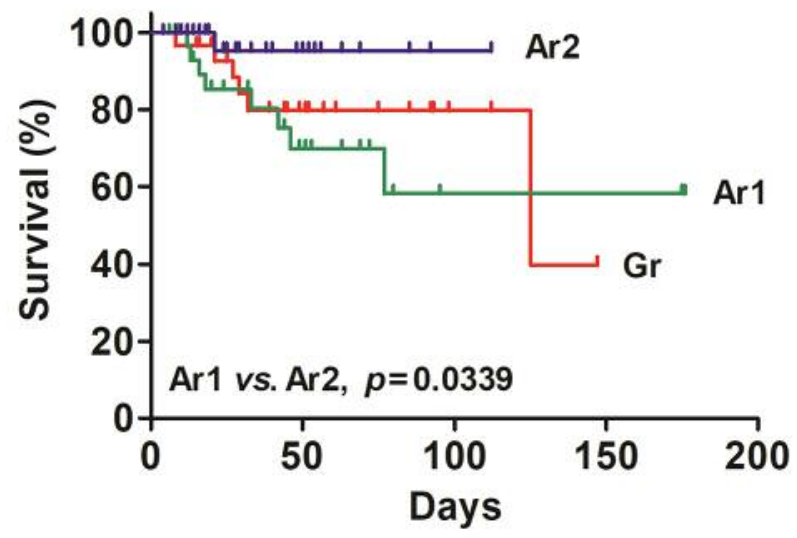

Figure 1. Catheter failure-free survival was estimated using the KaplanMeier method. The log-rank test was performed to compare survival distributions according to PICC.

formation were not considered. Large prospective studies, including patients of different ethnicities and having different cancers, are required to validate the results of the present study.

\section{Conclusion}

Synthetic polymer-coated PICC is safe and effective to administer anticancer chemotherapy with less catheter complications.

\section{Conflicts of Interest}

The Authors declare that they have no conflicts of interest.

\section{Authors' Contributions}

Y Takezawa wrote the initial draft of the manuscript and contributed to analysis and interpretation of data. K Izumi designed the study, contributed to analysis and interpretation of data, assisted in the preparation of the manuscript, and supervise all process. All other authors have contributed to data interpretation and critically reviewed the manuscript.

\section{References}

1 Bertoglio S, Faccini B, Lalli L, Cafiero F and Bruzzi P: Peripherally inserted central catheters (PICCs) in cancer patients under chemotherapy: A prospective study on the incidence of complications and overall failures. J Surg Oncol 113: 708-714, 2016. PMID: 27020965. DOI: 10.1002/jso.24220

2 McGee DC and Gould MK: Preventing complications of central venous catheterization. N Engl J Med 348: 1123-1133, 2003. PMID: 12646670. DOI: 10.1056/NEJMra011883 
3 Lv Y, Hou Y, Pan B, Ma Y, Li P, Yu L, Xu D, Song J, Shang H, Wang $\mathrm{H}$ and Tian Y: Risk associated with central catheters for malignant tumor patients: a systematic review and meta-analysis. Oncotarget 9: 12376-12388, 2018. PMID: 29552318. DOI: 10.18632/oncotarget. 24212

4 Morano SG, Latagliata R, Girmenia C, Massaro F, Berneschi P, Guerriero A, Giampaoletti M, Sammarco A, Annechini G, Fama A, Di Rocco A, Chistolini A, Micozzi A, Molica M, Barberi W, Minotti C, Brunetti GA, Breccia M, Cartoni C, Capria S, Rosa G, Alimena G and Foà R: Catheter-associated bloodstream infections and thrombotic risk in hematologic patients with peripherally inserted central catheters (PICC). Support Care Cancer 23: 3289-3295, 2015. PMID: 25910751. DOI: 10.1007/ s00520-015-2740-7

5 Saijo F, Odaka Y, Mutoh M, Katayose Y and Tokumura H: A novel technique of axillary vein puncture involving peripherally inserted central venous catheters for a small basilic vein. J Vasc Access 19: 311-315, 2018. PMID: 29592529. DOI: 10.1177/ 1129729818757974

6 Paquet F, Boucher LM, Valenti D and Lindsay R: Impact of arm selection on the incidence of PICC complications: results of a randomized controlled trial. J Vasc Access 18: 408-414, 2017. PMID: 28665468. DOI: 10.5301/jva.5000738

7 Fallouh N, McGuirk HM, Flanders SA and Chopra V: Peripherally inserted central catheter-associated deep vein thrombosis: A narrative review. Am J Med 128: 722-738, 2015. PMID: 25697969. DOI: 10.1016/j.amjmed.2015.01.027
8 Leung A, Heal C, Perera M and Pretorius C: A systematic review of patient-related risk factors for catheter-related thrombosis. J Thromb Thrombolysis 40: 363-373, 2015. PMID: 25680892. DOI: $10.1007 / \mathrm{s} 11239-015-1175-9$

9 Kang JR, Long LH, Yan SW, Wei WW, Jun HZ and Chen W: Peripherally inserted central catheter-related vein thrombosis in patients with lung cancer. Clin Appl Thromb Hemost 23: 181186, 2017. PMID: 26207019. DOI: 10.1177/1076029615595880

10 Curto-Garcia N, Garcia-Suarez J, Callejas Chavarria M, Gil Fernández JJ, Martín Guerrero Y, Magro Mazo E, Marcellini Antonio S, Juárez LM, Gutierrez I, Arranz JJ, Montalvo I, Elvira C, Domínguez P, Díaz MT and Burgaleta C: A team-based multidisciplinary approach to managing peripherally inserted central catheter complications in high-risk haematological patients: a prospective study. Support Care Cancer 24: 93-101, 2016. PMID: 25935657. DOI: 10.1007/s00520-015-2754-1

Received February 7, 2019

Revised February 25, 2019

Accepted February 26, 2019 